\title{
Cold atmospheric plasma with self-organized patterns for cancer therapy
}

\author{
Zhitong Chen* \\ Department of Mechanical and Aerospace Engineering, The George Washington University, Washington, DC 20052, USA
}

\begin{abstract}
This paper overviews the formation of the self-organization plasma structures and potential applications in cancer therapy. Representative experimental evidence of self-organized patterns (SOPs) in diverse types of plasma discharges in contact with liquid electrodes are reviewed. SOP plasma in contact with liquids show marked sensitivity of pattern formation at the interphase to broad range of controlling parameters, such as driving current, electrolyte conductivity, gas length, gas species, and gas flow rate. Several studies suggest that reactive species formed in self-organized plasma structure are effective against cancer cells both in vitro and vivo.
\end{abstract}

\section{Introduction}

Self-organization is complex and fascinating phenomena commonly observed in both natural and technological contexts, within diverse varieties of physics, chemistry and biology [1]. Different types of self-organization phenomena have been reported in a wide range of plasmas, such as dielectric barrier discharge [2], high frequency discharge [3], gas flow stabilized discharges [4], resistively stabilized discharged [5], and discharges with liquid electrodes [6]. The SOPs are significantly different among these discharges, which typically occur in the anode or cathode. Recently, number of plasma discharges in which one or two electrodes are in the liquid phase have been studied referring to applications ranging from water decontamination and activation, to nanoparticle and materials synthesis, and medicine [7-12]. Therefore, SOP plasma is of interest not only from a fundamental point of view, as intrinsic and fascinating characteristics of nature, but also from practical standpoint of current and emerging technologies. The main objective of this review is to summarize fundamental scientific aspects of plasma self-organization phenomena and to describe their potential applications in cancer therapy.

\section{Self-organization in plasma discharges}

SOPs in plasma discharges interacting with liquid surfaces are more pronounced, in particular, when conductive liquid acts as a cathode or an anode. Plasma discharges interacting with liquids can be distinguished among those within lower aspect-ratios (e.g. jets) and those within high aspect-ratio conjurations (e.g. planar discharges). Plasma on liquids shows strong sensitivity to self-organization events and a wider range of intervening parameters. SOPs include squaretextures, square-lattices, square/hexagonal superlattices, hollowhexagonal, multi-armed spirals, and rotating-wheels patterns [13]. Therefore, the formation of these depends on various parameters such as driving current, electrolyte conductivity, gap length, gas species, and so on.

Due to the large diversity of physical and chemical phenomena involved plasma processes related to self-organization are rather complicated for their theoretical understanding [14,15]. Theoretical models of plasma discharge in ambient gas, including the most basic ones, admit multiple solutions existing for the same discharge current. One of these solutions is in the simplest case one-dimensional, which describes states with a uniform distribution of current over the electrode surface. The other existing solutions are in all the cases multidimensional and describe modes with different configurations of cathode spots. The hypothesis of multiple solutions existence was proposed in 1963 for arc-cathode interaction and derived in 1988 for plasma discharge [14]. Currently, solutions describing diffuse and spot modes of current transfer to cathodes of high-pressure arc discharges have been computed under different conditions by different research groups and validated by extensive comparison with the experiment [16,17].

\section{Effect of experimental conditions on SOPs}

Current and voltage play important roles in generating SOPs in glow discharge. Figure 1 shows current-voltage characteristics and optical photographs discharge patterns above the liquid corresponding to the specific current/voltage conditions [18]. The whole currentvoltage characteristic can be divided into four specific stages: a single

${ }^{\star}$ Correspondence to: Chen Z, Department of Mechanical and Aerospace Engineering, The George Washington University, Washington, DC 20052, USA, E-mail: zhitongchen@gwu.edu

Special Issue: Nanotechnology: Challenges and Perspectives in Medicine

Dr. Federica Valentini

Department of Sciences and Chemical Technologies

Tor Vergata University

Italy

Maurizio Talamo

Professor

Department of Enterprise Engineering

Italy

Key words: cold atmospheric plasma, self-organized patterns, plasma medicine, cancer therapy, reactive oxygen species, reactive nitrogen species

Received: February 18, 2019; Accepted: March 11, 2019; Published: March 14 2019 


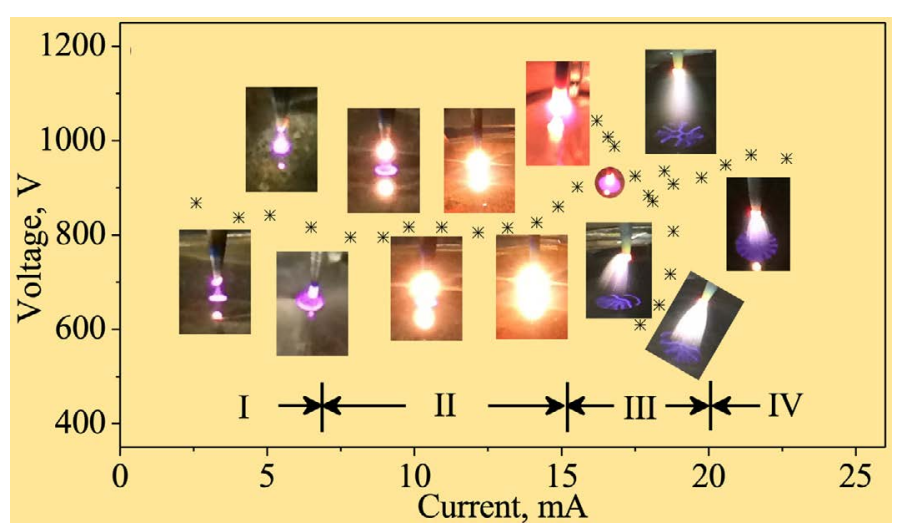

Figure 1. Optical photographs of the discharge patterns above the liquid during the activation process. Stratification of plasma jet to a large number of thin current filaments is clearly visible at lower photograph of the panel; fine complex patters are also formed on the surface of liquid media. Four discharge stages could be clearly determined [18].

filament following the initial corona discharge (stage I), drastically enhanced heat radiation (stage II), an unstable state (transition stage, stage III), and the multi-filament pattern (stage IV). At stage IV, the discharge stabilizes at the multi-filament stage and stretches out to large number of discharge filaments at liquid media surface (selforganized patterns). Complex shapes consisting of radial and confocal lines of different density can be produced, and some elements of radial/ axial symmetry can be observed. On the other hand, Miao, et al. and Wilson have reported the anode spot had a small circular shape when the discharge current was $10 \mathrm{~mA}[19,20]$. While the anode spot turned into a ring-like structure with current increasing from 15 to $25 \mathrm{~mA}$. Further increasing the discharge current caused the near anode plasma self-organization. Moreover, due to change in equivalent resistance of the liquid electrode, a drop of plasma temperature with decreasing current is reason for voltage decreasing with increasing conductivity [21]. Meanwhile, the continuous operation of the plasma, causing the gradual acidification of the liquid and increase of electrolyte conductivity, leads to increasingly constricted anode self-organized patterns. Apart from current and electrolyte conductivity, gap lengths also affect self-organized patterns of atmospheric glow discharge on liquids electrodes [22]. In liquid solution anodes, SOPs appear when the gap length is large. SOPs will disappear when the gap length decreases. Similar results also can be found in Figure 2 (upper level). In addition to the discharge current, electrolyte conductivity, and gap length, the gas species of plasma discharges produce a diverse range of effects on the SOPs. No gas (air) flow and nitrogen $\left(\mathrm{N}_{2}\right)$ flow from the outside appear the same ring-shaped anode pattern, while oxygen $\left(\mathrm{O}_{2}\right)$ flow from outside shows that the anode spot is moved and its shape gradually changes [23]. A variety of patterns are observed using $\mathrm{O}_{2}$ sheath flow, and the patterns change their shape randomly. Therefore, the existence of $\mathrm{O}_{2}$ strongly affects the anode pattern structure. Wilson, et al. elucidate that the ring-like structure is observed in air, $\mathrm{N}_{2}$, and a $\mathrm{N}_{2}-\mathrm{H}_{2}$ mixture, but not in pure $\mathrm{N}_{2}, \mathrm{H}_{2}$, or He. Electronegative gases generally capture free electrons and form negative ions in a discharge, which may result into changing the number of electrons near the liquid electrode surface. In the process of discharge using a liquid electrode, the gas (such as $\mathrm{H}_{2}, \mathrm{Cl}_{\text {or }} \mathrm{O}_{2}$ ) will appear at the water surface due to the electrolysis. Although the effect of the gas on mechanisms of pattern formation are not yet completely understood, the existence of $\mathrm{O}_{2}$ strongly influences the anode pattern structure.

\section{Potential applications for cancer therapy}

Plasma medicine is an innovative and emerging field of interdisciplinary research that combines biology, chemistry, physics, and medicine. Plasma is known for the generation of charged particles, electronically excited atoms, reactive oxygen species (ROS), and reactive nitrogen species (RNS). ROS and RNS, combined or independently, are known to promote cell proliferation as well as cell death. Additionally, extreme amounts of reactive species may lead to the damage of deoxyribose nucleic acid (DNA), proteins, lipids, senescence, and induce apoptosis [24-26]. Figure 2 (upper level) shows that atmospheric discharge between a metal pin cathode and saline solution anode with different air gap lengths $(2-10 \mathrm{~mm})$ was considered for investigating SOP plasma at the liquid anode surface [22]. With gap increasing, the discharge current decreases while discharge voltage increases. Optical emission spectrum by the SOP plasma discharge above saline solution taken using UV- visible-NIR at $4 \mathrm{~mm}$ and $6 \mathrm{~mm}$ air gap length were shown in Figure 2 (middle level). The dominant species of the spectra in this figure were $\mathrm{NO}$ or $\mathrm{N}_{2}$ lines $(258,267$, 337, and $357 \mathrm{~nm}), \mathrm{OH}(309 \mathrm{~nm}), \mathrm{N}_{2}^{+}(391 \mathrm{~nm})$, and $\mathrm{O}(777 \mathrm{~nm})$. The presence of ROS and RNS leads to the formation of short- and long-lived species, which are important for the anti-cancer properties. Short-lived radicals or specials includes superoxide $\left(\mathrm{O}^{2-}\right)$, nitrite $(\mathrm{NO})$, atomic oxygen $(\mathrm{O})$, ozone $\left(\mathrm{O}_{3}\right)$, hydroxyl radical $(\bullet \mathrm{OH})$, singlet delta oxygen $\left(\mathrm{SOD}, \mathrm{O}_{2}(1 \Delta \mathrm{g})\right.$ ), peroxynitrite $\left(\mathrm{ONOO}^{-}\right)$, and so on [25,27]. Long-lived species include hydrogen peroxide $\left(\mathrm{H}_{2} \mathrm{O}_{2}\right)$ and nitrite $\left(\mathrm{NO}^{2-}\right.$ ). Transport of ROS/RNS across the plasma/liquid interface is affected by SOP. As such modification of saline solution by discharge is affected

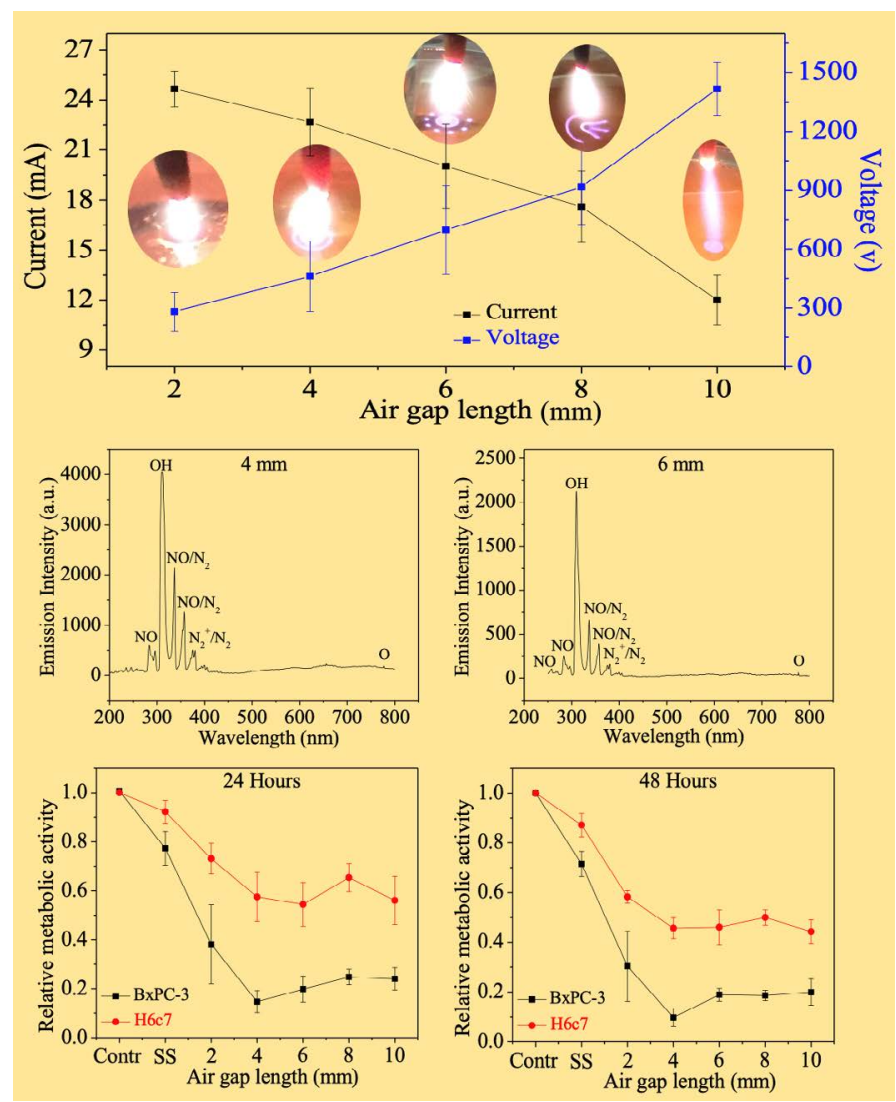

Figure 2. Atmospheric discharge with different air gap lengths was considered for investigating SOP plasma at the liquid anode surface. SOP plasma-activated saline solutions were applied to human pancreas adenocarcinoma cancer cells line (BxPC-3) and human pancreatic duct epithelial normal cells line (H6c7). Upper level: current-voltage dependence for different air gap lengths with optical photographs of the self-organized interface patterns. Middle level: optical emission spectrum by the SOP plasma discharge above saline solution taken using UV- visible-NIR at $4 \mathrm{~mm}$ and $6 \mathrm{~mm}$ air gap length. Lower level: effects of SOP plasma-activated saline solutions on viability of the BxPC-3 and H6c7 after 24- and 48-hours' incubation, respectively [22] 
and controlled by SOP at the plasma-liquid interface. Figure 2 (lower level) shows SOP plasma-activated saline solutions applied to human pancreas adenocarcinoma cancer cells line $\left(\mathrm{BxPC}^{-3}\right)$ and human pancreatic duct epithelial normal cells line $\left(\mathrm{H}_{6} \mathrm{c}_{7}\right)$, and SOP plasmaactivated saline solution affecting cancer and normal pancreatic cells in a selective manner. SOP plasma-activated saline solutions might affect these non-oncogene dependencies in the context of a transformed genotype and might result in a synthetic lethal interaction and the selective death of $\mathrm{BxPC}^{-3}$ cancer cell compared to $\mathrm{H}_{6} \mathrm{c}_{7}$ normal cell. Plasma with SOP activating saline solutions have more effect on cancer cells. The trend of pancreatic normal and cancer cells can be attributed to the trend of ROS and RNS concentration with different air gap distances. On the other hand, plasma with SOP-induced intracellular generation of ROS induced apoptosis in $\mathrm{BcPC}^{-3}$ cancer cells might be orchestrated by the synergistic effects of both extrinsic and intrinsic pathways [28]. The results indicate the cytotoxicity of plasma-activated saline solution is specific to pancreatic adenocarcinoma cancer cells.

On the other hand, different SOPs appear dielectric-barrier discharges (DBD) plasma, and DBD plasma has been applied to cancer therapy. SOP plasma kills Melanoma skin cancer cells through necrosis at higher treatment doses through necrosis at higher treatment doses, which are still below the threshold of damaging healthy tissues [29]. Very low doses of plasma initiate apoptosis of Melanoma skin cancer cells where no cell necrosis was observed. Vandamme, et al. indicated that plasma treatment is safe for mice, and a dramatical U87 bioluminescence decrease associated with a reduction of tumor volume in U87 glioma-bearing mice after 5-day treatment [29]. Panngon, et al. investigated preferential killing of human lung cancer cells (H460) and normal cells (MRC5 and L132) by SOP plasma [30]. The timedependent cell death implies that the cell death induced by plasma was not necrotic. The apoptosis is a complex process including at least two main processes: intrinsic pathway and extrinsic pathway [31]. The mitochondria have a main role in deciding a cell fate in intrinsic pathway, and death receptors have a key role in mediating external signals to caspase8 in extrinsic pathway. Unregulated mRNA expression of both Caspase [8] and Bax genes in H460, under plasma treatment, implies the activation of both intrinsic and extrinsic pathways. Bax is a Bcl-2 family related to singling of mitochondrial membrane in the process of early apoptosis.

SOP plasma can treat more different media such as water, saline solution, Ringer's solution, Dulbecco's Modified Eagle's Medium (DMEM), Foetal Bovine Serum (FBS), and so on, which can be used as an anti-tumor media. Currently, some papers proposed plasmaactivated media as a new chemotherapy. Boehm, et al. elucidates the exposure of FBS to atmospheric plasma resulting into the generation of relatively long-lived products such as hydrogen peroxide $\left(\mathrm{H}_{2} \mathrm{O}_{2}\right)$, which are biologically active and have demonstrated anti-microbial and cytotoxic activity [32]. Nguyen, et al. reports anti-cancer efficacy of plasma dissolved in a liquid and liquid plasma in heterogeneous cancer cells [33]. Tanaka, et al. investigated that plasma activated Ringer's solution for clinical applications [34]. The chemical composition of plasma-activated media has shown a strong decrease of the solution's $\mathrm{pH}$, increases in oxidation/reduction potential and the generation of ROS and RNS. SOP plasma-activated media, containing ROS and RNS, has been reported to initiate the apoptotic cascade in mammalian cells with selectively higher cytotoxicity in cancerous cell lines. ROS is known cytotoxic agent and has been reported as one of the main mediators of cold plasma cytotoxicity, cell growth is inhibited at concentrations lower than those in cultures supplemented with commercially available $\mathrm{H}_{2} \mathrm{O}_{2}[35,36]$. ROS is not the only toxic species present in the plasma-activated solutions, but other cytotoxic components increased with plasma exposure time in line with $\mathrm{H}_{2} \mathrm{O}_{2}$ [37]. The formation of RNS has been proposed to be the cause of synergistic anti-microbial effects of these compounds and may also account for enhanced cytotoxicity. SOP plasma-generated reactive species result into increasing in intracellular ROS: low concentration can stimulate cell proliferation or cytokine release but can cause DNA damage; and higher concentrations induce cell cycle arrest and trigger apoptosis [38]. The generation of intracellular ROS inducing apoptosis has been postulated as the pathway through which SOP plasma may be a promising therapy for tumor treatment, it also bears the risks of causing oxidative stress-induced genetic and epigenetic alterations involved in carcinogenesis [39].

\section{Conclusions}

This review demonstrates experimental investigations of the formation of the self-organization. Approaches for the control of SOP formation, either by suppressing or enhancing them, include driving current, electrolyte conductivity, gas length, gas species, and imposed gas flow. Moreover, convincing evidences of SOP plasma towards cancer therapy have been accumulated. Various aspects of reactive species that can be produced by SOP plasma were studied worldwide, which are effective against cancer cells both in vitro and vivo. The cancer-inhibiting media has acquired a pronounced cancer-depression activity towards cancer cells. Fundamental studies in the area of SOP plasma for cancer therapy are further required, and challenges of this area is rapidly growing. Scientists will pay more attentions on SOP plasma for cancer therapy not only because the payoffs are substantial, but because it is one of few remaining areas of modern technology whose application in medicine has been barely explored.

\section{References}

1. Trelles JP (2013) Formation of self-organized anode patterns in arc discharge simulations. Plasma Sources Science and Technology 22: 025017.

2. Kogelschatz U (2002) Filamentary, patterned, and diffuse barrier discharges. IEEE Transactions on plasma science 30: 1400-1408.

3. Shi J, Liu D, Kong MG (2006) Plasma stability control using dielectric barriers in radio-frequency atmospheric pressure glow discharges. Applied phys lett 89: 081502.

4. Akishev Y (2001) The influence of electrode geometry and gas flow on corona-to-glow and glow-to-spark threshold currents in air. J Phys D: Applied Phys 34: 2875.

5. Laroussi M, Alexeff I, Richardson JP, Dyer FF (2002) The resistive barrier discharge. IEEE Transactions on Plasma Science 30: 158-159.

6. Verreycken T, Bruggeman P, Leys C (2009) Anode pattern formation in atmospheric pressure air glow discharges with water anode. J Applied Phys 105: 083312.

7. Locke B, Sato M, Sunka P, Hoffmann M, Chang JS (2006) Electrohydraulic discharge and nonthermal plasma for water treatment. Industrial \& engineering chemistry research 45: 882-905.

8. Chen Z, Cheng X, Lin L, Keidar M (2016) Cold atmospheric plasma discharged in water and its potential use in cancer therapy. J Phys D: Applied Phys 50: 015208.

9. Chen Z, Lin L, Cheng X, Gjika E, Keidar, M (2016) Treatment of gastric cancer cells with nonthermal atmospheric plasma generated in water. Biointerphases 11: 031010.

10. Kaneko T, Takahashi S, Hatakeyama R (2012) Control of nanoparticle synthesis using physical and chemical dynamics of gas-liquid interfacial non-equilibrium plasmas. Plasma Physics and Controlled Fusion 54: 124027.

11. Weltmann KD (2010) Atmospheric-pressure plasma sources: Prospective tools for plasma medicine. Pure and Applied Chemistry 82: 1223-1237.

12. Keidar M (2018) A prospectus on innovations in the plasma treatment of cancer Physics of Plasmas 25: 083504.

13. Dong L (2012) Collective vibration of discharge current filaments in a self-organized pattern within a dielectric barrier discharge. Phys Rev E 85: 066403. 
14. Trelles JP (2017) Finite element methods for arc discharge simulation. Plasma Processes and Polymers 14: 1600092.

15. Hartmann P, Reyes JC, Kostadinova EG, Matthews LS (2019) Self-diffusion in two-dimensional quasimagnetized rotating dusty plasmas. Phys Rev E 99: 013203. [Crossref]

16. Benilov M (2008) Bifurcations of current transfer through a collisional sheath with ionization and self-organization on glow cathodes. Phys Rev E 77: 036408

17. Trelles JP (2016) Pattern formation and self-organization in plasmas interacting with surfaces. J Phys D: Applied Phys 49, 393002 (2016).

18. Chen Z, Zhang S, Levchenko I, Beilis II, Keidar M (2017) In vitro demonstration of cancer inhibiting properties from stratified self-organized plasma-liquid interface. Scientific reports 7: 12163 .

19. Miao SY (2008) Conical DC discharge in ambient air using water as an electrode. IEEE Transactions on Plasma Science 36: 126-130.

20. Wilson A (2008) Self-rotating dc atmospheric-pressure discharge over a water-surface electrode: regimes of operation. Plasma Sources Science and Technology 17: 045001.

21. Bruggeman P (2008) Characteristics of atmospheric pressure air discharges with a liquid cathode and a metal anode. Plasma Sources Science and Technology 17: 025012.

22. Chen Z (2018) Selective treatment of pancreatic cancer cells by plasma-activated saline solutions. IEEE Transactions on Radiation and Plasma Medical Sciences 2: 116-120.

23. Shirai N, Uchida S, Tochikubo F (2014) Influence of oxygen gas on characteristics of self-organized luminous pattern formation observed in an atmospheric dc glow discharge using a liquid electrode. Plasma Sources Science and Technology 23: 054010.

24. Chen Z, Lin L, Cheng X, Gjika E, Keidar M (2016) Effects of cold atmospheric plasma generated in deionized water in cell cancer therapy. Plasma Processes and Polymers 13: 1151-1156.

25. Chen Z, Simonyan H, Cheng X, Gjika E (2017) A Novel Micro Cold Atmospheric Plasma Device for Glioblastoma Both In Vitro and In Vivo. Cancers (Basel) 9. [Crossref]

26. Keidar M (2015) Plasma for cancer treatment. Plasma Sources Science and Technology 24: 033001 .
27. Xu RG, Chen Z, Keidar M, Leng Y (2018) The impact of radicals in cold atmospheric plasma on the structural modification of gap junction: a reactive molecular dynamics study. International Journal of Smart and Nano Materials 1-12.

28. Zhang R, Humphreys I, Sahu RP, Shi Y, Srivastava SK (2008) In vitro and in vivo induction of apoptosis by capsaicin in pancreatic cancer cells is mediated through ROS generation and mitochondrial death pathway. Apoptosis 13: 1465-1478.

29. Vandamme M (2010) Antitumor effect of plasma treatment on U87 glioma xenografts: preliminary results. Plasma processes and polymers 7: 264-273.

30. Panngom K (2013) Preferential killing of human lung cancer cell lines with mitochondrial dysfunction by nonthermal dielectric barrier discharge plasma. Cell death \& disease 4: $\mathrm{e} 642$.

31. Hoentsch M, von Woedtke T, Weltmann KD, Nebe JB (2011) Time-dependent effects of low-temperature atmospheric-pressure argon plasma on epithelial cell attachment, viability and tight junction formation in vitro. J Phys D: Applied Phys 45: 025206.

32. Boehm D, Heslin C, Cullen PJ, Bourke P (2016) Cytotoxic and mutagenic potential of solutions exposed to cold atmospheric plasma. Scientific reports $6: 21464$.

33. Nguyen NH, Park HJ, Yang SS, Choi KS, Lee JS (2016) Anti-cancer efficacy of nonthermal plasma dissolved in a liquid, liquid plasma in heterogeneous cancer cells. Scientific reports 6: 29020

34. Tanaka H (2016) Non-thermal atmospheric pressure plasma activates lactate in Ringer's solution for anti-tumor effects. Scientific reports 6: 36282.

35. Haertel B, von Woedtke T, Weltmann KD, Lindequist, U (2014) Non-thermal atmospheric-pressure plasma possible application in wound healing. Biomolecules \& therapeutics 22: 477 .

36. Li W (2019) Cold atmospheric plasma and iron oxide-based magnetic nanoparticles for synergetic lung cancer therapy. Free Radical Biology and Medicine 130: 71-81.

37. Chen Z (2018) Micro-sized cold atmospheric plasma source for brain and breast cancer treatment. Plasma Medicine 8: 203-215.

38. Sensenig R (2011) Retracted article: Non-thermal plasma induces apoptosis in melanoma cells via production of intracellular reactive oxygen species. Ann Biomed Eng 39: 674-687.

39. Ishaq M (2014) Atmospheric gas plasma-induced ROS production activates TNFASK1 pathway for the induction of melanoma cancer cell apoptosis. Molecular biology of the cell 25: 1523-1531.

Copyright: (C2019 Chen Z. This is an open-access article distributed under the terms of the Creative Commons Attribution License, which permits unrestricted use, distribution, and reproduction in any medium, provided the original author and source are credited. 